\title{
Análisis de la producción y consumo de carne en la provincia de Chimborazo, Ecuador.
}

\author{
Analysis of meat production and consumption in the province of \\ Chimborazo, Ecuador
}

Jacqueline Carolina Sánchez Lunavictoria. ${ }^{1}$ \& Carlos Augusto Delgado Rodríguez. ${ }^{2}$

Recibido: 14-03-2021 / Revisado: 21-03-2021 /Aceptado: 11-04-2021/ Publicado: 05-05-2021

\begin{abstract}
.
DOI: https://doi.org/10.33262/concienciadigital.v4i2.1.1709

Introduction. Meat consumption trends; the way it is produced and marketed is changing worldwide. In this sense, Ecuador has not been the exception, and particularly the province of Chimborazo where both the production and consumption of this food has undergone changes. On the other hand, the issue of health, environmental awareness are some of the factors that have influenced the consumption of this protein and that also merit an analysis. The objective of the research proposes a theoretical approach to the analysis of the production and consumption of meat in the province of Chimborazo in order to serve as a baseline and contribution for future investigations that wish to deepen the subject, as well as guide future commercial decisions and of production within the province. Methodology. The research design is qualitative-quantitative and crosssectional. The methods used were the historical-logical, inductive-deductive, analyticalsynthetic. Results. Among the most significant results, it can be highlighted that almost half of the cattle destined for meat production are found on the Coast, while dairy cattle are concentrated in the Sierra. Chimborazo represents only $6 \%$ of livestock production compared to the other provinces. Conclusions. One of the main conclusions is that factors such as health, price, product presentation and ecology affect the consumption of red meat.
\end{abstract}

\footnotetext{
${ }^{1}$ Escuela Superior Politécnica de Chimborazo, Facultad de Administración de Empresas. Riobamba, Ecuador. carolina.sanchez@espoch.edu.ec, https://orcid.org/0000-0002-3059-2823

2 Escuela Superior Politécnica de Chimborazo, Facultad de Administración de Empresas. Riobamba, Ecuador. carlos.delgado@espoch.edu.ec, http://orcid.org/0000-0002-9822-4322
} 
Keywords: meat production and consumption, trending changes, shift in demand.

\section{Resumen.}

Introducción. Las tendencias de consumo de carne; la manera de producirla y comercializarla está cambiando a nivel mundial. En este sentido Ecuador no ha sido la excepción, y particularmente la provincia de Chimborazo donde tanto la producción y consumo de este alimento ha experimentado cambios. Por otra parte, el tema de la salud, la conciencia ambientalista son algunos de los factores que han incidido en el consumo de esta proteína y que amerita también un análisis. El objetivo de la investigación plantea una aproximación teórica al análisis de la producción y consumo de carne en la provincia de Chimborazo a fin de que sirva como línea base y aporte para futuras investigaciones que deseen profundizar en la temática, así como guía futuras decisiones comerciales y de producción dentro de la provincia. Metodología. El diseño de la investigación es de tipo cualicuantitativo de corte transversal. Los métodos empleados fueron el histórico-lógico, inductivo -deductivo, analítico sintético. Resultados. Entre los resultados más significativos se pueden destacar que casi la mitad de ganado destinado a la producción de carne se encuentra en la Costa mientras que el ganado lechero se concentra en la Sierra. Chimborazo representa apenas el $6 \%$ de producción de cabezas de ganado con respecto a las otras provincias. Conclusiones. Una de las principales conclusiones es que factores como la salud, el precio, la presentación del producto y la ecología, inciden en el consumo de carne roja.

Palabras claves: producción y consumo de carne, cambios tendenciales, desplazamiento de demanda.

\section{Introducción.}

En el contexto mundial en el que factores como el incremento de la población, cambios en la dieta y estilos de vida causan una mayor ingesta de proteína animal; se estima que la demanda mundial de productos cárnicos se incremente a un ritmo anual de $1.3 \%$ entre 2007 y 2050, valor superior al crecimiento anual estimado de $1.1 \%$ para el total de la producción agropecuaria en igual lapso. (ESPAE, 2016).

Se proyecta además que el mayor impulso a la demanda de cárnicos provenga de los países en vías de desarrollo. Al mismo tiempo, se evidencia un cambio en la importancia de distintos tipos de producción de proteína animal, que aumenta para animales como aves y cerdos. Afectaciones futuras sobre la industria penderán también de las percepciones del público sobre la relación entre carnes rojas y la salud humana. Elementos como la creciente conciencia medio ambiental, nuevas tendencias de consumo como la vegana inciden en los patrones de consumo y producción. (ESPAE, 2016).

Para Heiman et al. (2001), la demanda y consumo de carne es influenciado por el comportamiento del precio, educación e ingreso de los consumidores. Coincidiendo con 
este autor se encuentra el planteamiento de Bernanke y Frank (2007) quienes afirman que la demanda está determinada por las preferencias personales y por el poder adquisitivo de los individuos.

En suma, existen tres grandes grupos de producciones de carne animal: de bovino, cerdo y aves. Las proporciones de su consumo están influenciadas por los factores antes mencionados y su producción va de la mano tanto con el consumo a nivel local y como con sus relaciones comerciales a nivel global.

La producción de carne en el mundo muestra un importante grado de concentración por país, con los diez principales productores representando más del $50 \%$ de la producción mundial, y sólo los 3 primeros aportan alrededor del $40 \%$ del total global.

Son diversos y múltiples los factores que influyen sobre la producción de alimentos, pero una de las consecuencias de esta tendiente disparidad, es el constante crecimiento de la población, donde la producción de los alimentos no alcanza a satisfacer los requerimientos necesarios (FAO, 2006; citado por Ayala, 2018).

En el Ecuador el consumo de productos derivados de la carne es uno de los sostenes de la alimentación. Destacando los embutidos cuyo consumo ha ido aumentando día a día.

Chimborazo forma parte de las ocho provincias donde se concentra el mayor consumo de productos cárnicos en el Ecuador, contando con 228500 especies en pie para la producción de carne de res (INEC, 2018).

El presente trabajo se traza como objetivo, realizar un análisis descriptivo de la producción y consumo de carne en la provincia de Chimborazo, Ecuador.

\section{Metodología.}

Para llevar a cabo esta investigación, se empleó el método histórico- lógico que incluyó la revisión bibliográfica y documental de fuentes especializadas en la materia. A partir de la recopilación de información ya existente sobre estas temáticas en revistas y artículos científicos y otros trabajos académicos, se realizó un análisis y síntesis proporcionando una visión sobre el estado de la producción y el consumo de carne en la actualidad. Además, se utilizaron métodos como el inductivo - deductivo y técnicas matemáticas en la construcción de gráficos a partir del estudio de bases de datos del Banco Central de Ecuador (BCE) y el Ministerio de la Agricultura y Ganadería (MINAG).

\section{Resultados.}

\section{Producción de carne en la provincia de Chimborazo, Ecuador.}

En el periodo 2014 - 2019, la producción de ganado vacuno en el Ecuador abarcó el 66\% de la producción ganadera total. El ganado porcino y ovino un promedio de $21 \%$ y $7 \%$ 
respectivamente, la diferencia se encuentra distribuida en el resto de especies: asnal, caballar, mular y caprino. (INEC, 2019). Ver tabla 1.

Tabla 1. Número de cabezas de ganado

\begin{tabular}{llllllll}
\hline Años & Vacuno & Porcino & Ovino & Asnal & Caballar & Mular & Caprino \\
\hline $\mathbf{2 0 1 4}$ & 4.579 .374 & 1.910 .319 & 619.366 & 84.785 & 283.714 & 98.259 & 20.793 \\
\hline $\mathbf{2 0 1 5}$ & 4.115 .213 & 1.637 .662 & 506.696 & 59.070 & 223.352 & 88.123 & 27.102 \\
\hline $\mathbf{2 0 1 6}$ & 4.127 .311 & 1.141 .244 & 478.486 & 49.960 & 219.134 & 79.287 & 36.379 \\
\hline $\mathbf{2 0 1 7}$ & 4.190 .611 & 1.115 .473 & 390.120 & 49.727 & 209.990 & 80.111 & 39.583 \\
\hline $\mathbf{2 0 1 8}$ & 4.056 .796 & 1.283 .338 & 355.897 & 47.035 & 192.833 & 73.681 & 21.745 \\
\hline $\mathbf{2 0 1 9}$ & 4.306 .244 & 1.162 .685 & 464.644 & 61.155 & 196.886 & 83.008 & 28.391
\end{tabular}

Fuente: Instituto Nacional de Estadísticas y Censos INEC- ESPAC 2014- 2019.

En porcentajes, la distribución se representa en la figura 1.

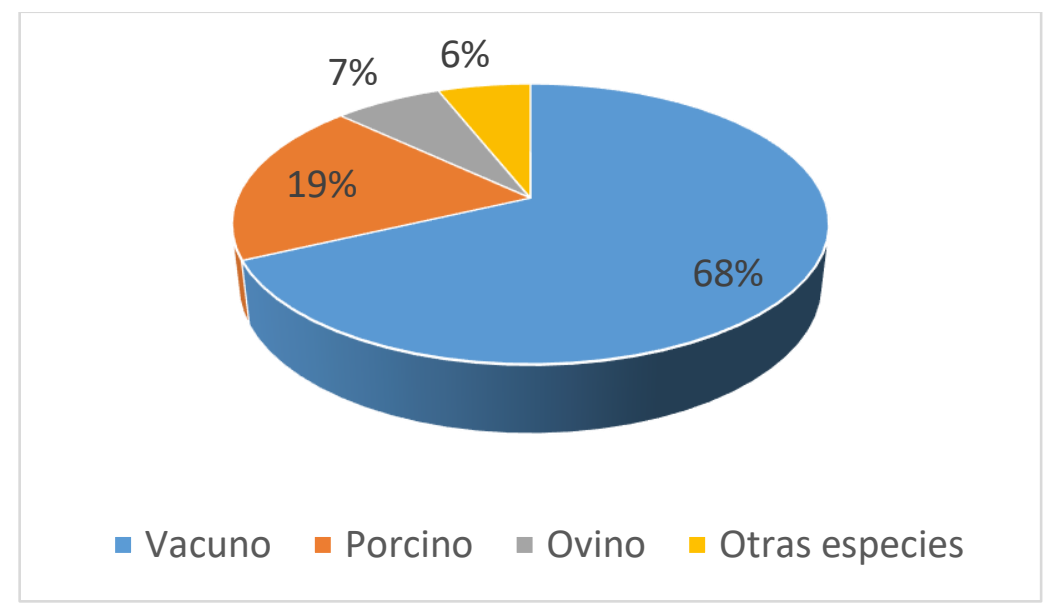

Figura 1. Participación de especies de ganado Ecuador 2019.

Fuente: Instituto Nacional de Estadísticas y Censos INEC- ESPAC 2014- 2019.

Casi la mitad del ganado de carne se encuentra en la Costa (principalmente en Manabí, Azuay y Guayas), mientras el ganado lechero se concentra en la Sierra (más de tres cuartas partes). Las importaciones de animales vivos en 2019 fueron menores que años precedentes, provenientes principalmente de EE.UU., Perú y Brasil.

En el año 2018, la provincia de Manabí registró la mayor producción de cabezas de ganado con un 19\% del total en el país, mientras que Azuay (8\%), Guayas (7\%), Cotopaxi (7\%), El Oro (6\%) y Chimborazo $(6 \%)$ le siguen entre las principales productoras de cabezas de ganado. 
Por otra parte, la producción neta ${ }^{2}$ de la carne de pollo, cerdo y res ha experimentado un leve crecimiento alcanzando en el año 2018 una producción neta de 763.984 toneladas, entre otros factores, por el incremento de la población del país. El crecimiento de la población mundial, sumado al aumento del consumo per cápita de fuentes de proteína animal en las economías emergentes o en desarrollo generan un desafío de magnitud para toda la cadena de valor de alimentos.

\section{Tendencia creciente de producción neta de carnes con respecto a la población.}

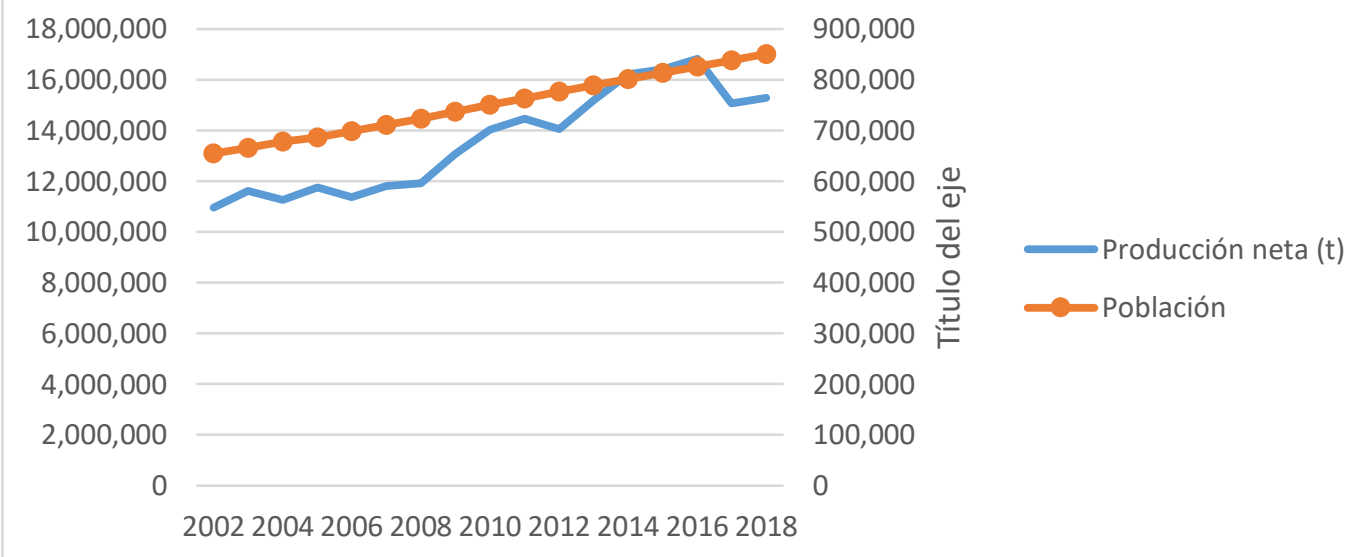

Figura 2: Tendencia creciente de producción neta de carnes con respecto a la población. Fuente: Elaboración propia a partir de bases de datos del (MAG, 2018).

Así mismo, las importaciones de carne se han incrementado en los últimos 4 años, siendo los principales proveedores EE.UU., Chile y Uruguay. Mientras que las exportaciones han experimentado un decrecimiento total, siendo casi nulo. Según la ESPAE (2016), en la industria local la declaración del Ecuador libre de fiebre aftosa con vacunación generó mucha expectativa en torno a la exportación de cárnicos. Elementos como el tamaño del hato ganadero, la débil industrialización, el crecimiento de consumo de aves donde la industrialización ha crecido en los últimos años, y la baja rentabilidad, influyen para que estas expectativas de exportación no se concreten. Otras debilidades como el estado sanitario de los establecimientos para el faenamiento, la inseguridad, e infraestructura inadecuada, se suman a esta preocupación. (ESPAE 2016),

La población bovina de la Costa se redujo a una tasa anual de $3.2 \%$ en el período 2011 2014, en comparación a un incremento de 3.3\% al año en la Sierra y de $1.7 \%$ en la Amazonía. Se cree que entre los factores que han constituido una limitante para el desarrollo de la producción local están los costos de producción elevados.

Además, la superficie plantada de pastos cultivados para el ganado ha registrado un ligero decrecimiento a partir del año 2015, alcanzando en el año 2018 las 47.619 hectáreas.

\footnotetext{
${ }^{2}$ Producción Bruta menos impurezas, humedad, pérdidas, desperdicios, destino a semillas, alimento animal, industria no alimenticia, subproductos y coeficientes de transformación a producto homologado. No es comparable por transformación de producto bruto a producto homologado (Fuente: MINAG).
} 
Algunos factores asociados a este decrecimiento son la mala calidad de pastos debido a la escasa disponibilidad de agua en el sector; razón por la cual, su producción es destinada para autoconsumo, mientras que las demás especies para la venta y consumo. (Jaramillo, et al., 2017).

La producción de cabezas de ganado en Chimborazo ha disminuido considerablemente en los últimos años, registrando en 2018 un total de 401.038 cabezas de ganado menos con respecto al 2015.

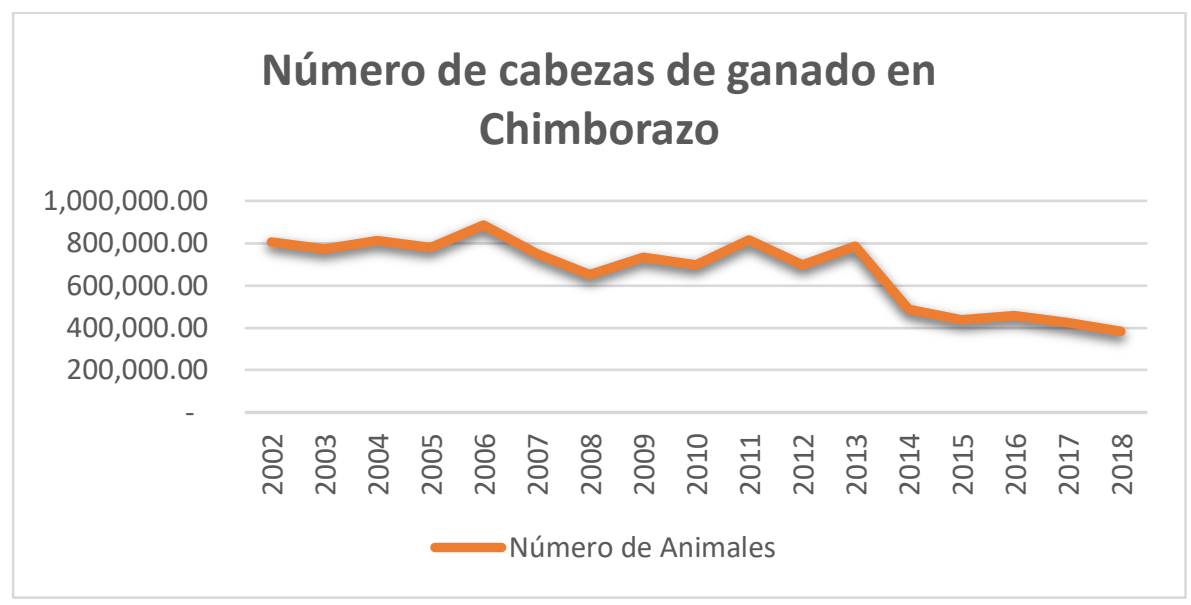

Figura 3: Número de cabezas de ganado en Chimborazo 2002-2018.

Fuente: Elaboración propia a partir de bases de datos del (MAG,2018)

El caso de Chimborazo no se aleja demasiado del comportamiento a nivel país. La tendencia a decrecer se ha manifestado desde hace ya varios años, en especial en el 2014 donde se vio una baja importante en las cabezas de ganado. Es importante recalcar que esta provincia no tiene un gran peso dentro del sector agropecuario, teniendo un porcentaje de participación en el mismo de no más del $6 \%$ y ha disminuido en la última década, especialmente en lo que ha ganado respecta.

En Chimborazo se toman toda una serie de iniciativas para el mejoramiento de la calidad de la producción de carne. Existen proyectos que pretenden contribuir a mejorar la economía y seguridad alimentaria de las familias campesinas, a través del incremento de la producción y comercialización de productos agropecuarios y en especial la ganadería de carne. Uno de ellos se implementó en el año 2014 en el cantón Guano con la introducción de toretes de la raza, con la finalidad de aportar en el desarrollo de la producción ganadera y por otra mejorar las condiciones de vida de sus habitantes.

La mejora genética de los animales destinados a la producción cárnica se basa en obtener animales de peso ideal, en menor tiempo y con altos índices de calidad de carne, sin comprometer la inversión a lo largo de su desarrollo productivo (Jaramillo et al., 2017). 
Es necesario el incremento de iniciativas que contribuyan al mejoramiento de la calidad de la producción de carne, pues a pesar de que la producción pecuaria es variada, no es tecnificada, razón por la cual los rendimientos son bajos.

\section{Consumo de carne en la provincia de Chimborazo, Ecuador.}

En Ecuador, destaca el crecimiento en el consumo de la carne de aves hasta convertirse en la más importante desde 2011, mientras el consumo de carne bovina y de cerdo crecía a menor ritmo. En el sector agropecuario predomina el ganado vacuno con un total de 4.1 millones de cabezas (cifra que ha mermado con respecto a años anteriores), seguido por el ganado porcino con 1.3 millones de cabezas.

Al comparar las cifras de consumo ecuatorianas con los demás países latinoamericanos, se puede observar que existen oportunidades importantes de crecimiento en la industria, como es el caso de Perú, líder en consumo de carne de pollo con $46.66 \mathrm{Kg}$ per cápita, seguido de Argentina $44 \mathrm{Kg}$, Bolivia $43 \mathrm{Kg}$, Brasil y Panamá $42 \mathrm{Kg}$ (WATT Global Media, 2018). Mientras que para la carne de cerdo el primero en consumo per cápita es Chile $(22.8 \mathrm{Kg}$ ), seguido de México $(17 \mathrm{Kg}$ ) y Panamá $(15.9 \mathrm{Kg})$. (Pig Improvement Company, 2018). Y en el caso de la carne de res los mayores consumidores son Uruguay, Argentina, Paraguay y Brasil con 46, 41, 26 y $24 \mathrm{Kg}$ per cápita respectivamente (FIRA, 2017). Estas cifras resultan muy prometedoras para la industria cárnica en Ecuador y sugieren un potencial importante de crecimiento del sector, siempre que exista la aceptación por parte de los consumidores y los mismos cuenten con ingresos que les permitan adquirir proteína de origen animal.

Existe una cantidad limitada de estudios en Ecuador que permitan comprender claramente la tendencia del consumo de cárnicos, entre ellos se menciona la investigación de Castillo y Jaramillo (2012), citado en Rodríguez et al. (2019), quienes analizaron la tendencia de consumo de cárnicos en los clientes de una empresa productora y comercializadora de carne en Guayaquil, Ecuador y concluyeron que la percepción de los encuestados sobre la carne de cerdo es que resulta ser la menos saludable, sin embargo, resaltaron el hecho que es la más exquisita para el consumo. Para el caso del pollo, resultó ser la alternativa cárnica más económica, siendo la más demandada y rápida de preparar. Para 2019, el consumo de carne de pollo en el país fue de $30,43 \mathrm{~kg}$ por persona al año; es decir, $16 \%$ más alto que en 2018. (INEC,2018).

En la provincia de Chimborazo, el consumo de carne se comporta de manera similar al del país. En el estudio realizado por Ramos et al. (2015), sobre la calidad del producto, conocimiento y percepción de los expendedores de carne en el cantón Riobamba, se determinó que, de acuerdo al tipo de carne, se venden aproximadamente un 22,5\% de carnes blancas, $45,2 \%$ de carnes rojas y $31,6 \%$ de ambas. Siendo las carnes rojas las más demandadas.

Por otro lado, y desde el punto de vista del consumidor final, en un estudio más actualizado realizado por Gualán (2019), se concluye que los consumidores han disminuido la compra de carne de res debido al alto precio con el que se comercializa. En 
lugar de este producto los consumidores han optado por el consumo de la carne de pollo, puesto que cuentan con un menor precio. Al respecto del consumo de la carne de pollo; en el país existen 1.819 granjas avícolas, que generan empleo directo para alrededor de 32.000 personas, pero si se le agrega la cadena de maíz, soya, balanceados y cerdo las fuentes de empleo llegan a 220.000. (MAG, 2019).

Chimborazo de manera general sigue las tendencias de producción y consumo a nivel país, pero en un por ciento considerablemente menor. Por otra parte, la producción en su mayoría se realiza para el consumo local no tanto así para la exportación.

En este orden ideas se puede inferir que el consumidor final está cambiando su perspectiva sobre la carne roja y esto ha ido modificando los patrones de consumo tanto de Ecuador como de Chimborazo. El cambio en las exigencias del consumidor es mayor en cuanto a propiedades, información, salud, entre otras. Existe una creencia generalizada sobre la repercusión del consumo de carne (especialmente carne roja) y problemas de salud. De todo esto se pudo concluir que hubo un desplazamiento en las tendencias de consumo en los últimos 10 años.

\section{Conclusiones.}

- Según nuevos hábitos y condicionamientos culturales, las personas asocian los problemas de salud como causa derivada del consumo de carne, por lo que prefieren disminuir su consumo.

- La carne de res es considerada de mejor calidad si se encuentra dentro de un empaque.

- La higiene es un factor que genera confianza en los clientes.

- La carne empacada tiene un precio más alto, pero existe un porcentaje de población que estaría dispuesto a pagar el precio a cambio de tener un producto de calidad.

- La carne de cerdo es una opción de consumo después de la carne de res.

- El pollo, resultó ser la alternativa cárnica más económica, por tal motivo es la más demandada.

- Los nuevos paradigmas ambientales y de conciencia sostenible incidirán en el consumo de carne a futuro.

- La reducción de hato ganadero, el estado sanitario de los establecimientos para el faenamiento, la inseguridad, e infraestructura inadecuada, la débil industrialización son unos de los factores que reducen las expectativas de exportación de carne.

\section{Referencias bibliográficas.}

Ayala Vargas, C. (2018). Importancia nutricional de la carne. Revista de Investigación e Innovación Agropecuaria y de Recursos Naturales, 5(ESPECIAL) 
Bernanke, B., y R. Frank. 2007. Oferta y Demanda. In: Principios de Economía, Tercera Edición. McGraw Hill. pp: 62-90

Castillo, J., y Jaramillo, H. (2012). Caracterización de la tendencia del consumo de los Productos Cárnicos de los Clientes de la Coorporación Fernández y Propuesta de Estrategias de Fidelización. Universidad Politécnica Salesiana, Sede Guayaquil.

ESPAE (2016). Estudios Industriales. Orientación Estratégica para la toma de decisiones. Industria de Ganadería de carne. Recuperado de: https://www.espae.espol.edu.ec/wpcontent/uploads/2016/12/industriaganaderia.pdf

FAO (2006) . El estado de la inseguridad alimentaria en el mundo, Roma, Italia.

Gualán, W. (2019). Estudio de las preferencias de consumo de productos cárnicos para la empresa Holstein, ciudad de Riobamba. Tesis de pregrado. Espoch. Ecuador

Heiman, A. et al. (2001). Incorporating family interactions and socioeconomics variables into family production functions: the case of demand of meats. Agrobussines, 17 pp.455-468

INEC (2018). Boletín técnico No-01-2018-ESPAC. Encuesta de Superficie y Producción Agropecuaria Continua, 2018. Quito, Ecuador.

INEC (2019). Instituto Nacional de Estadísticas y Censos INEC- ESPAC 2014- 2019.

Jaramillo, I. et al., (2017). "Costo de producción y precio justo de la carne bovina en el Ecuador". Observatorio de la Economía Latinoamericana. Recuperado de: http://www.eumed.net/cursecon/ ecolat/ec /2017/produccion-carne-bovina.html

MAG (2018). Sistema de Información pública y agropecuaria.

MAG (2019). Ecuador celebra a la carne de pollo. Recuperado de: https://www.agricultura.gob.ec/ecuador-celebra-a-la-carne-de-pollo/

Pig Improvement Company (2018). Análisis de la industria porcina en Latinoamérica. Recuperado de: https://www.pic.com/

Ramos, M. et al. (2015). Conocimiento de los expendedores de carne sobre el faenamiento y comercialización, a través de análisis implicativo y descriptivo. Estudio de caso camal Riobamba Chimborazo - Ecuador. European Scientific Journal November, 11, (32)2 pp. 402-417

Rodríguez, D. et al., (2019). Técnicas cuantitativas de investigación de mercados aplicadas al consumo de carne en la generación millennial de la ciudad de Cuenca Ecuador. Revista Espacios, Vol. 40 (32), pp. 20 
WATT Global Media. (2018). Empresas Líderes. Industria Avícola. Recuperado de http://www.industriaavicola-digital.com/201804/index.php\#/40

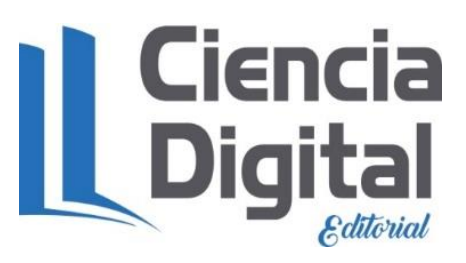




\section{PARA CITAR EL ARTÍCULO INDEXADO.}

Sánchez Lunavictoria, J. C., \& Delgado Rodríguez, C. A. (2021). Análisis de la producción y consumo de carne en la provincia de Chimborazo, Ecuador. ConcienciaDigital, 4(2.1), 81-91. https://doi.org/10.33262/concienciadigital.v4i2.1.1709

\section{\Ciencia}

El artículo que se publica es de exclusiva responsabilidad de los autores y no necesariamente reflejan el pensamiento de la Revista Ciencia Digital.

El artículo queda en propiedad de la revista y, por tanto, su publicación parcial y/o total en otro medio tiene que ser autorizado por el director de la Revista Ciencia Digital.

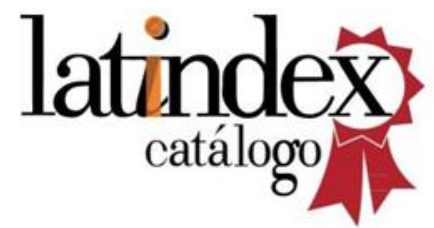

